\title{
CUARENTA AÑOS DE LA LEY ELECTORAL PARA EL PARLAMENTO DE CATALUNYA
}

\author{
JAUME MAGRE FERRÁN \\ Profesor Titular de Ciencia Política \\ Universidad de Barcelona \\ Director de la Fundació Carles Pi i Sunyer d'estudis autonòmics i locals
}

TRC, núm. 45, 2020, pp. 379-401

ISSN 1139-5583

\section{SUMARIO}

I. Introducción. II. El origen del sistema electoral: el Estatuto de Autonomía de 1979. III. El segundo momento: de la estabilidad bipartidista al Estatuto del 2006. IV. La Comisión de expertos del año 2007 y la ponencia parlamentaria del año 2010. V. Conclusiones.

\section{INTRODUCCIÓN}

El Parlamento de Catalunya ha sido elegido en doce ocasiones — desde 1980 a 2020 - a pesar de que la Comunidad Autónoma catalana carece, cuarenta años después de su constitución, de ley electoral. Su ausencia ha sido cubierta en el ámbito autonómico con las disposiciones transitorias del Estatuto de Autonomía de 1979 y por la Ley 5/1984, de 5 de marzo, por la que se adapta la normativa electoral general para las elecciones al Parlamento de 1984, cuyo único artículo reitera las previsiones de la Disposición Transitoria cuarta del Estatuto; a su vez, la norma que ha colmado las lagunas autonómicas ha sido la LOREG, y lo fue el Decreto-Ley de 1977 en las dos primeras elecciones autonómicas en Catalunya ${ }^{1}$. Para las primeras elecciones, la Disposición Transitoria cuarta se limitó a fijar como circunscripciones electorales las cuatro provincias de Barcelona, Girona, Lleida y Tarragona, así como un prorrateo de escaños entre ellas, y asignar las

1 Presno, M., «Nuevos Estatutos de autonomía con viejos sistemas electorales», Revista d'estudis autonòmics i federals, $\mathrm{n}^{\circ}$ 5, 2007, p. 125. 
competencias de organización y justicia electorales a las juntas provinciales electorales y en la Audiencia Territorial de Barcelona. En todos los demás aspectos, se remitió a las normas vigentes para las elecciones al Congreso de los Diputados de las Cortes Generales. El nuevo Estatuto de autonomía vigente desde 2006 establece que el régimen electoral será regulado por una ley del Parlamento aprobada por una mayoría cualificada de dos terceras partes de los diputados y mantiene vigente el contenido de la Disposición Transitoria cuarta del estatuto de autonomía de 1979 (DT $2^{a}$ EA 2006). A pesar de los muchos años transcurridos, pues, la prevista ley electoral no se ha llegado a aprobar y Catalunya se ha convertido en la única comunidad autónoma que no tiene una ley electoral propia.

La provisionalidad del modelo de sistema electoral ha provocado una considerable literatura académica sobre la cuestión. Los primeros trabajos tienen un carácter de comparación autonómica ${ }^{2}$, pero muy rápidamente aparecen los estudios sobre posibles reformas de la ley electoral y las propuestas de sistema electoral catalán ${ }^{3}$. A pesar de que los primeros intentos datan de mediados de los años noventa ${ }^{4}$, los resultados de las elecciones autonómicas de 1999 y 2003 desencadenan un intenso debate y un volumen importante de propuestas: desde la aplicación del sistema alemán ${ }^{5}$, la adaptación del sistema regional de Hessen y Escocia ${ }^{6}$, la aplicación de los números primos en el reparto de escaños ${ }^{7}$, la ponderación de prorrateo con variables territoriales ${ }^{8}$, el reparto biproporcional de los escaños ${ }^{9}$ hasta la aplicación territorial de las directrices del Informe Roca ${ }^{10}$. En cambio, son pocos los artículos que se centran en los efectos y posibles explicaciones del actual sistema electoral ${ }^{11}$. Entre éstos, sobresale el estudio de Montero y Lago en el que

2 Montero, J. R., Llera, F. y Torcal, M., «Sistemas electorales en España: una recapitulación», Revista Española de Investigaciones Sociológicas, $\mathrm{n}^{\circ}$ 58, 1992, pp. 7-56; LLERA, F. «Los rendimientos de los sistemas electorales de las Comunidades Autónomas: el predominio del bipartidismo imperfecto», Revista Española de Investigaciones Sociológicas, $\mathrm{n}^{\circ}$ 82, pp.127-157.

3 VAllès, J. M. y CANALS, R., «Cap a una llei electoral catalana: elements i propostes per al desenvolupament de l'article 31 de l'Estatut», en VAlLÈs, J. M. (Ed.), Reforma electoral i coordenades polítiques. Els condicionants de la normativa electoral a Espanya i a Catalunya, Barcelona, Fundació Jaume Bofill, 1982.

$4 \mathrm{BoscH}, \mathrm{A} .$, El sistema electoral. Una proposta per al Parlament de Catalunya, Barcelona, Fundació Jaume Bofill, 1995 .

5 Bosch, A., «Una proposta de sistema electoral», en VV.AA., Reflexions al voltant de la Llei electoral catalana, Barcelona, Fundació Carles Pi i Sunyer, 2002.

$6 \mathrm{Bosch}$, A., Adaptació a Catalunya dels sistemes electorals regionals de Hessen i Escòcia, Barcelona, Institut d'Estudis Autonòmics (material no publicado), 2002.

7 CAPO, J., "Crítica i proposta per a un sistema electoral per a Catalunya, en VV.AA», Reflexions al voltant de la Llei electoral catalana, Barcelona, Fundació Carles Pi i Sunyer, 2002.

8 Magre, J. y Pont, À., «Una proposta de sistema electoral per a Catalunya», en VV.AA., Reflexions al voltant de la Llei electoral catalana, Barcelona, Fundació Carles Pi i Sunyer, 2002.

9 López Carmona, A., Ramírez González, V. y Delgado Márquez, B. «Una propuesta de sistema electoral para Cataluña: reparto biproporcional», Política y Sociedad, vol. 51, núm. 3, pp. 841-862.

10 BurgueÑo, J., «Reflexions i propostes al voltant del sistema electoral a Catalunya: consells comarcals, diputacions, parlament i representació a corts», Scripta Nova, no 7, 2003, pp. 133-156.

11 Magre, J., «Reflexions entorn del sistema electoral del Parlament de Catalunya: efectes, propostes i límits», Revista d'estudis autonòmics i federals, $\mathrm{n}^{\circ} 1,2005$, pp. 163-188. 
introducen la idea de la variación de las magnitudes de distrito o «efecto varianza» para dar explicación a las mayorías parlamentarias, más o menos artificiales, de $\mathrm{CiU}^{12}$. Y aun son menos las publicaciones que se centren en los modelos de sistema electoral que formulan las fuerzas políticas y, sobretodo, no se han estudiado el cambio y la estabilidad de todos ellos a lo largo de estos cuarenta años. Es lo que pretende este trabajo, hacer un recorrido de las distintas propuestas a través de cuatro décadas de sistema político catalán, para acabar mostrando que, aun siendo posible el pacto, la rigidez de la reforma del nuevo Estatuto lo ha imposibilitado.

El presente artículo se organiza a partir de los tres momentos en los que las formaciones políticas exponen sus modelos de sistema electoral, los dos primeros ligados a la elaboración de los Estatutos de Sau, el 1979, y el vigente, aprobado el año 2006. En ambos casos, hemos circunscrito el análisis a las propuestas de las formaciones políticas de Disposición Transitoria relativa al sistema electoral que se presentaron para incluir en el Estatuto de autonomía. El tercer momento tiene un impulso institucional y enmarca la Comisión de expertos creada por acuerdo de Gobierno el año 2007 y la ponencia parlamentaria que, en 2010, intentó — sin éxito — un acuerdo a partir del trabajo realizado por la Comisión ${ }^{13}$.

\section{EL ORIGEN DEL SISTEMA ELECTORAL: EL ESTATUTO DE AUTONOMÍA DE 1979}

El interés de estudiar las propuestas de la Comisión de los veinte ${ }^{14}$ y del Pleno de los parlamentarios ${ }^{15}$ es doble. En primer lugar, dejar constancia de la discusión y pacto de la Disposición Transitoria cuarta que tenía que servir para

12 Montero, J. R. y Lago, I., «Más votos y menos escaños: el impacto del sistema electoral en las elecciones autonómicas catalanas de 2003», Revista Española de Investigaciones Sociológicas, $\mathrm{n}^{\circ}$ 105, 2004, pp. 11-42.

13 En febrero de 2013 se creó una nueva ponencia parlamentaria que desarrolló su tarea hasta el 2015. La ponencia no arrancó la unanimidad de todos los grupos parlamentarios y además el texto pactado no incorporaba el sistema electoral. Se consiguió un acuerdo limitado a ciertos aspectos del régimen electoral, pero no las variables estructurales - como la circunscripción, la fórmula de reparto de los escaños, la barrera mínima, el tipo de candidatura, entre otras - del sistema electoral. Por estos dos motivos no incorporamos los trabajos de la ponencia en nuestra investigación.

14 La Comisión de los veinte es la comisión de representantes que elaboró el anteproyecto de Estatuto de autonomía de 1979. Ese anteproyecto fue preparado por una representación de veinte diputados y senadores de las Cortes españolas escogidos en las primeras elecciones democráticas tras el franquismo. Los miembros de la Comisión fueron Macià Alavedra (CDC), Miquel Roca Junyent (CDC), Laureano López Rodó (AP), Eduardo Martín Toval (PSC), Josep Andreu Abelló (PSC), Josep Maria Triginer (PSC), Josep Benet (independiente por el PSUC), Felip Solé Sabarís (PSC), Jaume Sobrequés (independiente por el PSC), Josep Subirats (PSC), Josep Sendra (CDC), Jordi Solé Tura (PSUC), Josep Solé Barberà (PSUC), Dolors Calvet (PSUC), Josep Verde Aldea (PSC), Marcel-lí Moreta (UCD), Anton Cañellas (CC-UCD), Joaquim Arana (ERC), Manel de Sárrega Gómez (UCD) i Carles Güell de Sentmenat (CC-UCD)

15 El 25 de junio de 1977, sólo diez días después de las elecciones, todos los diputados y senadores se constituyen en Asamblea de Parlamentarios. 
llevar a cabo las primeras elecciones al Parlamento de Catalunya y ha acabado siendo el sistema electoral al Parlamento de Catalunya durante más de cuarenta años. En segundo lugar, establecer los distintos sistemas electorales que se proponen para Catalunya. Se trata, en cierto modo, del «momento cero» a partir del cual se pueden medir los cambios de las distintas propuestas de las fuerzas políticas a lo largo de estos años.

\section{La Disposición Transitoria como solución de compromiso}

La tarde del 5 de noviembre de 1978, la Comisión de los veinte se disponía a entrar en la recta final de la redacción del Estatut de Sau y lo hizo abordando un tema capital: la Disposición Transitoria que debía regular las primeras elecciones al Parlamento de Catalunya.

El principal punto de coincidencia entre los ponentes era la incomodidad sino el rechazo a las provincias como posibles circunscripciones electorales, pero en el resto y, sobre todo en las alternativas, la distancia entre formaciones políticas era insalvable. La no aceptación de la estructura provincial había sido una de las características distintivas del movimiento catalanista y este sentimiento, como veremos, determinó la creación de un frente comarcalista entre las fuerzas políticas que formaban parte de la Comisión y contribuyó a que la mayor discordia en los debates fuera, precisamente, la idea de representación territorial. No en vano, la concreción de las circunscripciones electorales fue el punto más enmendado del Estatut. A lo largo de las sesiones del Pleno de los parlamentarios, se presentaron un total de nueve enmiendas ${ }^{16}$.

Siendo la dimensión territorial el principal punto de conflicto, pues, la pregunta a la que debemos dar respuesta es a la situación paradójica de que la mayoría de las fuerzas políticas coincidieran en el rechazo a la provincia como distrito electoral, pero, en cambio, el resultado final de la Disposición Transitoria cuarta del Estatuto de 1979 certificase, precisamente, la provincia como circunscripción.

Para intentar dar respuesta al pacto al que se llegó, es necesario establecer el origen de los distintos posicionamientos de los ponentes y, este sentido, se entrecruzan tres elementos que es indispensable de tener en cuenta:

a) En primer lugar, tal como se ha señalado, la falta de aceptación de la estructura provincial había sido uno de las características distintivas del movimiento catalanista ${ }^{17}$. La comarcalización de 1936 disfrutó durante la transición

16 Concretamente, dos de CDC y una de AP, ERC, PSC, PSUC, UCD y UC-DCC, diputado Cañellas, diputado Sala i Canadell (CDC) y senador Ferrer i Gironès (Entesa dels Catalans, a título individual). Vid. Sobrequés, J., L'Estatut de la Transició. L'Estatut de Sau (1978-79), Barcelona, Parlament de Catalunya, 2010.

17 El catalanismo contrapuso a la estructura provincial de los liberales y moderados españoles una organización territorial alternativa basada en las comarcas. A lo largo de toda la historia de los últimos siglos, los dos modelos de organización territorial, el provincial-uniformista, por un lado, y 
política de un consenso considerable y de una fuerte aceptación social. El mapa comarcal republicano se convirtió en un elemento iconográfico asumido por todos los actores políticos y sociales, porque simbolizaba y resumía un conjunto de ideales de libertad y de recuperación nacional ${ }^{18}$. Durante la confección de anteproyecto de Estatuto, el frente comarcalista estuvo integrado por Convergència Democràtica de Catalunya (CDC), Unió del Centre i la Democràcia Cristiana de Catalunya (UC-DCC), Esquerra Republicana de Catalunya (ERC) y, curiosamente, la Unión del Centro Democrático (UCD).

Este bloque pretendía, de manera más o menos homogénea, que cada comarca se constituyese en circunscripción electoral o, en el peor de los casos, si esto no era aceptado, que las provincias con menos población pudieran elegir un mínimo de diputados suficientemente elevado para compensar el predominio poblacional de la conurbación de Barcelona. En otras palabras, los partidos de centro (UCD y UC-DCC), de derecha (AP) y el principal partido nacionalista (CDC) rechazaban un sistema proporcional estricto, para evitar que la provincia de Barcelona tuviera un peso representativo excesivamente elevado en el resultado final de las elecciones ${ }^{19}$.

En este sentido, la propuesta más elaborada y que se mantuvo de manera recurrente hasta la aprobación del anteproyecto de Estatuto fue la alternativa liderada por UCD y UC-DCC, en la que proponían que el futuro Parlamento tuviera dos cámaras, en la Cámara Alta, estarían representadas todas las comarcas, fuera cual fuera su población, mientras que la Cámara Baja sería elegida por criterios proporcionales. La cámara de los diputados tendría entre noventa y seis y ciento cuarenta y cuatro miembros. Para elegirlos, las circunscripciones electorales coincidirán con las provincias, a excepción del municipio de Barcelona, que formaría una circunscripción aparte. Cada distrito electoral elegiría un número mínimo de diputados fijo, y el resto de escaños se distribuiría en proporción a la población. La UCD mostraba un interés por establecer un Parlamento bicameral y el papel político preponderante otorgado a la representación territorial, es decir, a la representación comarcal, se debía al deseo de evitar una posible victoria de los partidos de izquierda en las elecciones al Parlamento de Catalunya ${ }^{20}$.

autonomista-comarcalista, por otro, se han ido sucediendo de acuerdo con los avatares históricos. Así, por ejemplo, la recuperación de un cierto nivel autonómico con la Mancomunidad de Prat de la Riba supuso la iniciación de un proceso de comarcalización que quedó estancado por el retorno del sistema provincial en la dictadura de Primo de Rivera, que a su vez, dio paso a la desaparición de las provincias y a la implantación de las comarcas y veguerías en tiempos de la República y, aún, a la reintroducción de la provincia con el triunfo del franquismo. Este debate sirvió para abastecer al nacionalismo catalán de una imagen del territorio diverso y antagónico a lo establecido desde el Estado central.

18 Burgueño, J., Història de la divisió comarcal, Barcelona, Rafael Dalmau, editor, 2003, p. 121.

19 La UCD propuso incluso mantener las cinco circunscripciones del Estatuto de 1932, eligiendo un diputado cada cincuenta mil habitantes, con un mínimo de doce diputados por circunscripción (Sobrequés et alt., 1982, pp. 115-122).

20 Sobrequés, J. y Riera, S., L'Estatut d'Autonomia de Catalunya: bases documentals per a l'estudi del procés polític d'elaboració de l'Estatut d'Autonomia de Catalunya de 1979, Barcelona, Edicions 62, 1982, p. 152. 
Lógicamente, la composición ideológica de la Comisión explica que la primera postura fuese claramente derrotada.

Más allá de las distintas propuestas, es interesante hacer notar que el frente comarcalista estaba dispuesto, en última instancia, a aceptar la provincia como distrito electoral si el prorrateo primaba a las zonas más despobladas de Catalunya. Esta posición allanaba el camino a un posible pacto que tuviera a la provincia como ámbito territorial de representación.

En sentido contrario, la izquierda (Partit dels Socialistes de Catalunya (PSC), Partit Socialista Unificat de Catalunya (PSUC) y Entesa dels Catalans), que tenía su base electoral mayoritaria en Barcelona y su entorno metropolitano, defendía un sistema que garantizara el valor igual de cada sufragio. Añadían que la elección de uno o dos diputados por comarca convertiría el sistema en mayoritario, con independencia de la fórmula de reparto de escaños que se decidiera escoger. Los socialistas, de entrada, propusieron la veguería ${ }^{21}$ y el PSUC introdujo una propuesta insólita bien que muy interesante: que Catalunya fuera una circunscripción única. Era la mejor manera de rentabilizar el peso de la conurbación barcelonesa, donde los comunistas tenían el grueso más consistente de su electorado ${ }^{22}$.

b) En segundo lugar, los ponentes del anteproyecto de Estatuto de autonomía de 1979 no tenían más experiencia electoral — más allá de los comicios de 1977- que la de las elecciones generales y municipales que se habían llevado a cabo pocos meses antes, el mes de marzo y abril, respectivamente. Las posiciones estratégicas de los diferentes partidos quedaban limitadas a estas primeras elecciones en las que los resultados fueron muy similares: el Parido Socialista fue el partido vencedor con un treinta por ciento de los votos y la principal fuerza política en diecinueve comarcas; la UCD se alzó con la segunda posición y fue el partido más votado en quince. Convergencia democrática obtuvo un resultado mediocre, ganando en 7 comarcas, la gran mayoría en la provincia de Girona.

Hubo tres zonas de dominio partidista: la primera y segunda corona metropolitana se convirtieron en el feudo electoral en general de la Izquierda y, más concretamente, del Partit dels Socialistes. Atendiendo a la concentración poblacional en esta zona territorial, es comprensible que la izquierda, en su conjunto, obtuviera cerca del 50\% del voto en estas elecciones. A pesar de la evidente concentración del voto socialista en la conurbación de la ciudad de Barcelona, el PSC es la organización que muestra una distribución más armónica en el territorio. Por lo que se refiere a las dos otras fuerzas políticas - CDC y UCD — la primera

21 El catalanismo político se caracterizará por la reivindicación de la división comarcal (y no en veguerías) como demarcación alternativa a la provincial. No será hasta la II República, cuando sea suprimida la división provincial en Catalunya y el Gobierno de la Generalidad diseñe una división territorial en 38 comarcas y 9 veguerías, si bien apenas estuvo vigente durante dos años (1936-38).

22 Sobrequés, J. y Riera, S., op. cit., p. 143. 
agrupa su voto en las comarcas de Girona y, en cambio, la UCD obtiene un resultado inesperadamente alto en las provincias de Lleida y Tarragona.

El hecho que no existiese una serie temporal electoral y que la única información disponible fuese el de las primeras elecciones generales cuando el sistema de partidos aún estaba formándose, pudo llevar a conclusiones precipitadas. Así, la UCD que, en líneas generales, provenía de una cultura política muy poco favorable a la existencia administrativa de las comarcas, se convierte en valedora de la estructura territorial de base comarcal. Aunque es cierto que el interés por establecer un Parlamento bicameral y el papel político preponderante otorgado a la representación comarcal se debía al deseo de evitar una posible victoria de los partidos de izquierda en las elecciones al Parlamento de Catalunya, el haber conseguido ser el partido más votado en quince comarcas puede haber ayudado a la inclusión de la UCD en el frente comarcalista de la ponencia estatutaria. En lo que se refiere a los mediocres resultados de CDC la percepción de sus elites era muy distinta a la que surgía de los resultados electorales: tal y como publicaba en 1978 en un artículo periodístico Joan Vidal i Gayolà de CDC, «(...) Pero esta, a pesar de ser importante (el resultado de las elecciones generales de 1977), no es la única razón que ha movido a los partidos sucursalistas a oponerse a la representación comarcal. Si me permiten una valoración muy subjetiva del hecho, le diré que, para mí, tiene su origen en un sentimiento de impotencia, en la íntima convicción de la propia incapacidad para presentar candidaturas con sentido a todas las comarcas de Catalunya y como suponen que nosotros (CDC) sí tenemos posibilidad de tenerlos, nos acusan de oportunismo electoral» (Los sitios, 21 de noviembre de 1978$)^{23}$. Mirando atrás, el Sr. Vidal probablemente tenía razón: la capacidad de penetración territorial y la porosidad popular de Convergència durante los próximos veinticinco años será una de las claves para entender la permanencia de esta organización en el Gobierno de la Generalitat.

c) Finalmente, entre un importante grupo de miembros de la ponencia — la mayoría de las filas de los partidos de izquierda - existía la voluntad de realizar las primeras elecciones al Parlamento de Catalunya con la máxima celeridad posible. En palabras del diputado Jordi Solé Tura, «(...) todos los diputados presentes a la Comisión de los veinte, recordarán que se llegó a una solución que tenía como única ventaja de permitirnos ir hacia unas elecciones de una manera rápida, de una manera que nos evitáramos obstáculos burocráticos y, sobretodo, obstáculos que podían venir de instancias que nosotros no controlamos, de autoridades que no están en Catalunya» ${ }^{24}$. Se albergaban dudas sobre las trabas que pudieran aparecer si la solución asumida fuese tan compleja que alargase en el tiempo la puesta en marcha de las primeras elecciones al Parlamento. Así, por ejemplo, se consideraba que la adopción de la comarca como circunscripción comportaba un conjunto de dificultades 
técnicas, como la falta de censos y de juntas electorales, que la desaconsejaba. La solución, pues, era la aceptación de un solución simple y eficaz, como era la provincia y que además no interfería en la futura organización territorial de Catalunya, al decantase por una determinada opción institucional. En el fondo, existía la plena conciencia entre los ponentes de la Comisión que se estaba debatiendo el sistema electoral únicamente de las primeras elecciones al Parlamento de Catalunya y no el que estaría en activo durante más de cuarenta años.

En conclusión, las primeras elecciones deberían atenerse a las provincias, por la simple razón de que no se disponía de otra unidad territorial adecuada e indiscutida entre los distintos partidos políticos. La mayoría de los ponentes coincidían en el rechazo de la provincia como distrito electoral, pero no en cuál era la alternativa. En este sentido, la Comisión de los veinte y, posteriormente, el Pleno de los parlamentarios, demostraron que existían dos tipos de soluciones: unas que hacían hincapié en la comarca, buscando con ello toda una explicación y una cierta concepción de Catalunya, y otras que postulaban la circunscripción única como solución. Entre estos dos extremos, la provincia apareció como la alternativa más pragmática, rápida y funcional.

Una vez establecida la provincia como circunscripción electoral — por paradójico que pareciera al inicio de los trabajos de la Comisión- el prorrateo de los escaños y la fórmula de atribución de los diputados no concitaron una discusión tan viva. Los socialistas propusieron dos alternativas: a) elegir un número mínimo de diputados por provincia, aceptándola como circunscripción electoral provisional, hasta que el Parlamento de Catalunya elaborara la correspondiente ley electoral, y con la posibilidad de reconocer una quinta circunscripción, que sería Barcelona-ciudad, más un diputado por $\mathrm{X}$ habitantes) o b) no fijar ningún número mínimo de diputados y elegir a uno por cada cincuenta mil habitantes en Barcelona, y uno por cada cuarenta mil habitantes en las otras tres provincias ${ }^{25}$.

La alternativa finalmente adoptada fue, como es sabido, la segunda. El Parlamento quedaría constituido por 114 diputados (82 por Barcelona, 12 por Tarragona, 11 por Girona y 9 por Lleida). La preponderancia de la izquierda en la Comisión de los veinte se dejó sentir en esta cuestión porque, si bien se facilitaba ligeramente la representación de las provincias menos pobladas, no se establecía un mínimo de diputados por circunscripción, lo que las hubiera favorecido más y hubiera facilitado el voto favorable del frente comarcalista ${ }^{26}$. En este sentido, la provincia de Barcelona, que representaba el $78 \%$ de la población catalana, recibía el $72 \%$ de los escaños. Un prorrateo, pues, que infrarrepresentaba en seis puntos porcentuales la provincia de Barcelona.

Finalmente, el punto seis de la Disposición Transitoria establecía que «en todo lo que no esté previsto en el presente Estatuto serán de aplicación las normas 
electorales que rigieron las elecciones legislativas a diputados del Estado del 15 de junio de 1977», lo que equivalía a establecer, sin mencionarlo, el reparto de escaños según el sistema D’Hondt y una barrera mínima del 3\%.

Una vez cerrado el pacto en la Comisión de los veinte, el anteproyecto debía ser avalado por el Pleno de los parlamentarios. La presión de los partidos políticos, de la prensa y de la opinión pública, obligaron a los ponentes a reabrir la cuestión territorial. Las posiciones de inicio de cada una de las formaciones políticas aparecieron de nuevo: se evidenciaba el rechazo de todas las fuerzas políticas hacia la provincia como distrito electoral, algunas de ellas, principalmente de centro y de derecha, habían continuado presionando para conseguir el reconocimiento de las comarcas como circunscripción electoral, mientras que los socialistas propusieron la veguería y el PSUC recuperó el modelo de circunscripción única ${ }^{27}$.

Finalmente, el 29 de diciembre de 1978 se aprobó el Estatuto con una redacción de la Disposición Transitoria cuarta que se alejaba notablemente del pacto inicial de la Comisión de los veinte: el Estatuto que llegaría a Las Cortes tenía a la veguería como distrito electoral, el sistema de cociente y resto mayor como método de distribución de los escaños y una barrera mínima del 3\%. Una construcción que difería de manera sensible del anteproyecto de Estatuto y que sólo se explica por las presiones de la opinión pública y los medios de comunicación que fueron especialmente beligerantes respecto la adopción de la comarca como circunscripción. Pero, en fondo, el camino ya estaba allanado: los ponentes eran conscientes que el punto de encuentro era el de la Comisión de los veinte.

La negociación en el Congreso de los diputados retomó el modelo de esta Comisión, probablemente por ser fruto del primer pacto entre las fuerzas políticas catalanas, como por ser muy similar al régimen electoral de las elecciones generales. La Disposición Transitoria cuarta del Estatuto establece, en su apartado segundo, la utilización de la provincia como circunscripción electoral para las elecciones al Parlamento de Catalunya. De esta forma, los electores de las cuatro provincias catalanas eligen 135 diputados: los de Barcelona eligen uno cada 50.000 habitantes, con un máximo de 85 escaños en juego, mientras que los de las provincias de Girona, Lleida y Tarragona eligen un mínimo de seis diputados, más otro por cada 40.000 habitantes, fijándose, en la Disposición transitoria, 17 escaños en Girona, 15 en Lleida y 18 en Tarragona.

Desgraciadamente no existe material de archivo para poder establecer los términos de la negociación, pero lo que resulta evidente es la voluntad de limitar el peso de la circunscripción de Barcelona en la composición de la cámara catalana: si la relación entre población/escaños de la Disposición Transitoria pactada en la Comisión de los veinte era equilibrada, con una ligera desproporción a favor

27 Íbidem, p. 251. 
de las zonas menos pobladas del territorio, en la versión definitiva esta misma divergencia aumenta de manera significativa.

La desproporción en el caso de la circunscripción de Barcelona es evidente. Entre una y otra propuesta ésta aumenta en 10 puntos porcentuales. Los resultados electorales de las elecciones generales de 1979 marcaron, en cierta medida, el tablero de juego: la izquierda consiguió un resultado contundente en el distrito barcelonés y se quiso limitar el impacto de estas fuerzas políticas en las elecciones al Parlamento de Catalunya. Éste es el mismo principio rector que explica la propuesta de un umbral máximo de 85 escaños al que se somete a la circunscripción de Barcelona, con independencia del crecimiento demográfico futuro que pudiera tener. Por otro lado, con la asignación de un número de diputados mínimo tan elevado por circunscripción los negociadores se aseguraban el apoyo o, como mínimo, la no oposición por parte de las fuerzas comarcalistas que, como mal menor, estaban dispuestos a aceptar un prorrateo que desproporcionase la asignación de los escaños a favor de los distritos menos poblados.

\section{El «momento cero» de los modelos de sistema electoral de los partidos}

Más allá del pacto de la Disposición Transitoria quinta (posteriormente cuarta) la discusión en el Pleno de los parlamentarios también nos permite, a grandes trazos, dibujar los sistemas electorales que empiezan a aparecer en el horizonte político catalán. A partir de las enmiendas realizadas por los ponentes a la Disposición Transitoria, se pueden establecer los primeros modelos de sistema electoral de los diferentes partidos políticos. En cierta manera, se establece el modelo cero de los sistemas electorales de cada una de las formaciones.

Los puntos de encuentro de las diferentes propuestas son notables: en primer lugar, todas las formaciones apostaron por una formula proporcional de reparto de los escaños, ya sea el socorrido sistema D'Hondt o la fórmula del cociente y restos más elevados. Lo interesante es que todos los ponentes entendieron que el momento, en cierta medida, obligaba a un reparto proporcional de los diputados del Parlamento de Catalunya. Incluso las formaciones de tradición más conservadora se inclinaron por aceptar la legislación electoral estatal de forma supletoria e, indirectamente, un reparto proporcional de los escaños. En segundo lugar, ninguna de las organizaciones que formaron parte del Pleno de los parlamentarios puso en duda la necesaria existencia de una barrera mínima. También en esta ocasión, la duda sobre el número de partidos que decidirían concurrir a las primeras elecciones al Parlamento de Catalunya, pudo haber jugado a favor de la aceptación sin demasiados miramientos, de la normativa electoral supletoria. En tercer lugar, tampoco tiene presencia en el debate parlamentario la forma de las candidaturas. No hay ninguna mención expresa al voto preferencial, ni a una apertura de las listas electorales, así como tampoco la magnitud de la cámara, más allá de aproximaciones probabilísticas de algún ponente atrevido. En resumen, pues, el 
núcleo central del sistema electoral no era de un interés especial para los ponentes, más allá de la ya citada voluntad de un funcionamiento, más o menos, proporcional del modelo. El foco principal de discusión es, como ya lo hemos advertido en repetidas ocasiones, la circunscripción electoral. Y, más concretamente, el papel de la comarca.

Es lógico que el debate se centrara en este aspecto ya que la delimitación y la magnitud de los distritos electorales es la clave para la evaluación de los efectos políticos y para el estudio del rendimiento de los sistemas electorales, tal como lo han planteado, entre otros, Duverger ${ }^{28}, \operatorname{Rae}^{29}$, Sartori $^{30}$, Lijphart $^{31}$ y Nohlen ${ }^{32}$. Es conocida también la coincidencia de investigadores españoles en identificar la magnitud de los distritos como principal fuente de desigualdad y de desproporcionalidad del sistema electoral español ${ }^{33}$. Pero, no creo que ninguno de los ponentes tuviera en mente a Maurice Duverger o Douglas Rae en la discusión sobre la organización territorial de Catalunya. Al contrario, la comarca era, prácticamente, una cuestión de principio a la que era muy difícil renunciar, aún para las fuerzas no estrictamente nacionalistas. En efecto, el debate comarcalista sirvió para abastecer al nacionalismo catalán de una imagen del territorio diverso y antagónico a lo establecido desde el Estado central, que quedaba representado por la provincia y las formaciones políticas no podían sustraerse al imaginario colectivo respecto de las instituciones franquistas. La importancia ideológica otorgada de la comarca en el debate del sistema electoral es el que, probablemente, explica la convergencia de varios de los modelos en la familia de los sistemas de doble voto.

\section{EL SEGUNDO MOMENTO: DE LA ESTABILIDAD BIPARTIDISTA AL ESTATUTO DEL 2006}

El momento fundacional del sistema político catalán ha quedado establecido y las normas que deben regir las primeras elecciones al Parlamento de Catalunya, también. De manera global, el conjunto del sistema encuentra su apoyo en la

28 Duverger, M., Los partidos políticos, México, Fondo de Cultura Económica, 1965.

29 RAE, D., The political consequences of electoral laws, New Haven, Yale University, 1971.

30 Sartori, G., «La Leggi sulla influenza dei sistema elettorali», Rivista Italiana di Scienza Politica, $\mathrm{n}^{\circ}$ 14, 1984, pp. 3-40; SARTORI, G., «The influence of electoral systems: faulty laws or faulty method», en B. Grofman y A. Lijphart (eds.), Electoral laws and their consequences, Nova York, Agathon Press, 1986; SARTORI, G., Comparative Constitutional Engineering: An inquiry into Structures, incentives and Outcomes, Londres: MacMillan, 1994.

31 Lijphart, A., «The political consequences of electoral laws, 1945-1985», American Political Science Review, vol. 84, n 2, 1990, pp. 481-496.

32 Nohlen, D., Sistemas electorales del mundo, Madrid, Centro de Estudios Constitucionales, 1981.

33 VAllès, J. M., Reforma electoral $i$ coordenades polítiques. Els condicionants de la normativa electoral a Espanya i a Catalunya, Barcelona, Fundació Jaume Bofill, 1982; MonTero, J. R., Llera, F. y TorCaL, M., op. cit., pp. 7-56; LAGO, I., «Cleavages y umbrales: las consecuencias políticas de los sistemas electorales autonómicos, 1980-2000», Revista Española de Ciencia Política, n 7, 2002, pp. 131-158. 
legislación electoral española, es decir, en la Ley Orgánica de Régimen Electoral General, que actúa no sólo como norma supletoria, sino que comprende la mayor parte de elementos que configuran el conjunto de su particular régimen electoral. Tal y como ya lo hemos expuesto, los electores de las cuatro provincias catalanas eligen 135 diputados: los de Barcelona eligen uno cada 50.000 habitantes, con un máximo de 85 escaños en juego, mientras que los de las provincias de Girona, Lleida y Tarragona eligen un mínimo de seis diputados, más otro por cada 40.000 habitantes, fijándose, en la Disposición Transitoria, 17 escaños en Girona, 15 en Lleida y 18 en Tarragona. Y, del mismo modo que para el Congreso de Diputados, las listas son cerradas y bloqueadas, y la fórmula electoral aplicada para atribuir los escaños es el sistema de Hondt, es decir, una fórmula proporcional de la familia de la media más alta.

Este segundo momento (1980-2006) es el intervalo en el que el sistema de partidos catalán se estabiliza y queda estructurado como un particular bipartidismo definido por la alternancia del Partit dels Socialistas de Catalunya y CiU en la victoria, según el tipo de elecciones, entre las que destaca de manera clara la hegemonía de CiU en las autonómicas, especialmente hasta finales de los años 90, cuando consigue la mayoría absoluta de sufragios durante tres legislaturas consecutivas $^{34}$.

El arraigo territorial de la coalición CiU también es decisivo. En este sentido, si realizamos una media de los resultados de los diferentes partidos y la observamos en las cuarenta y una comarcas catalanas, la coalición CiU es la formación vencedora en todas ellas, excepción hecha del Baix Llobregat.

En términos de sufragio, estos resultados incontestables de CIU vienen acompañados de un conjunto de efectos del sistema electoral que benefician de manera sensible a esta formación en el momento de transformar los resultados electorales en escaños. La alteración que provoca el sistema electoral no es sobre la proporcionalidad ${ }^{35}$ sino que, si bien consigue una relación relativamente equilibrada entre votos y escaños de todos los partidos políticos, siempre aparece la excepción de la coalición CiU, que se beneficia de una evidente distorsión que oscila entre los 3,2 puntos y 6,5 puntos porcentuales, según las legislaturas. La

34 Pallarés, F., «Las elecciones autonómicas del 19 de octubre de 1999 en Cataluña», en J. Tornos (dir), Informe de las Comunidades Autónomas. 1999, Barcelona, Instituto de Derecho Público, 2000, p. 714; Pallarés, F. «Las elecciones autonómicas de 16 de noviembre de 2003 en Cataluña», en J. Tornos (dir), Informe de las Comunidades Autónomas. 2003, Barcelona, Instituto de Derecho Público, 2004, p. 781.

35 En este periodo de estabilidad del sistema de partidos, el sistema electoral catalán ha propiciado unos efectos notablemente proporcionales. De acuerdo con el índice de Rose, el grado de proporcionalidad ha oscilado entre el 90,1\% de las primeras elecciones autonómicas, hasta el máximo alcanzado en 2003 que fue de un $96,7 \%$. Las hipotéticas distorsiones creadas por el sistema electoral, pues, tienen poco que ver con el grado de proporcionalidad que origina. De hecho, al tratarse de los efectos de los sistemas electorales, una de las pocas leyes que se cumplen determina que el nivel de proporcionalidad aumenta a medida que lo hace la magnitud de la circunscripción. El número de diputados que se eligen en cada una de las cuatro circunscripciones electorales catalanas permite un alto nivel de proporcionalidad en cada una de ellas. 
experiencia electoral de los primeros veinte y cinco años (1980-2006) demuestra que esta distorsión de los resultados ha afectado siempre positivamente a una misma formación política, que no es otra que CiU.

Esta alteración que provoca el sistema electoral pasaría inadvertida si la distribución territorial de los partidos políticos fuera homogénea en las cuatro circunscripciones electorales, pero este no es el caso: la presencia de la coalición CiU en las provincias de Tarragona y, sobre todo, Lleida y Girona es abrumadora, ya que desde 1984 alcanza un porcentaje que siempre ha oscilado entre una tercera parte y la mitad de los votos válidos en la circunscripción de Tarragona y entre el $60 \%$ y la mitad en los dos últimos distritos. Por el contrario, en la circunscripción de Barcelona el intervalo se sitúa entre el $40 \%$ y una tercera parte de los votos válidos. Ligada con esta distribución del voto, característica del modelo electoral catalán, Montero y Lago proponen explicar el grado de distorsión —en este periodo - a partir de la varianza de la magnitud de las circunscripciones: si existe una correlación entre la magnitud de los distritos y alguna característica política destacada del electorado, los escaños se distribuyen de manera diferente con un sistema electoral que tiene una amplia varianza en la magnitud de las circunscripciones que con otro que no la tenga ${ }^{36}$. En el caso catalán, y ya que la competición electoral depende fundamentalmente del número de escaños en juego, un partido mayoritario que consigue sus mejores resultados en las áreas urbanas tiene que competir con un número superior de rivales que otro que lo hace en las rurales. De esta forma, las preferencias partidistas en los distritos rurales se convierten más fácilmente en representación parlamentaria efectiva que las de los urbanos.

Sea como sea, el sistema electoral catalán no ha sido especialmente perjudicial para ninguna formación política en concreto, pero, en cambio, sí ha sido beneficioso para la coalición CiU desde las primeras elecciones. Así las cosas y sabiendo que las leyes electorales no se modifican con facilidad, ya que corresponde cambiarlas a los ganadores bajo el sistema electoral vigente ${ }^{37}$, Convergencia no ha tenido ningún incentivo para afrontar una reforma electoral. En efecto, hasta la sexta legislatura CiU ha gobernado con el apoyo de la mayoría absoluta del Parlamento de Catalunya y con el sostén de, como mínimo, el 40\% del electorado catalán. Como resulta comprensible, en estas condiciones, la modificación del sistema electoral no estuvo en la agenda política ni del Gobierno de la Generalitat ni de Convergencia durante estos veinticinco años.

Los resultados que se producen a partir de la convocatoria de 1999 suponen una cierta recomposición del sistema catalán de partidos que implica un escenario de igualdad entre las dos principales formaciones políticas - CiU y PSC — en las elecciones autonómicas y un incremento de la competencia provocado por el

36 Montero, J. R. y Lago, I., op. cit., pp. 31-34.

37 Cox, G., La coordinación estratégica de los sistemas electorales del mundo, Barcelona, Gedisa, 2004, p. 37. 
ascenso de fuerzas políticas tradicionalmente menores, desde el punto de vista electoral. Este nuevo escenario también ha comportado que, por dos veces consecutivas, la formación política que ha conseguido un mayor número de votos populares no sea la que ha obtenido un mayor número de diputados en el Parlamento de Catalunya. Este rendimiento del sistema electoral, favoreció la reconsideración del modelo de sistema electoral de los principales partidos políticos. A este escenario, hay que añadir dos circunstancias más. En primer lugar, teniendo en cuenta que el cambio de un sistema electoral es más probable a medida que crece el número efectivo de partidos ${ }^{38}$, el escenario político que se abre a partir de las elecciones de 1999 permite intuir que este éste podía haber sido un momento propicio para el cambio. En este sentido, el índice de Laakso y Taagepera, que pone en relación el número de partidos y su fuerza relativa, muestra que las elecciones de 2003 suponen el techo de la serie temporal respecto a esta variable. En segundo lugar, las convocatorias electorales de 1999 y 2003 provocaron unos resultados tan ajustados que las principales fuerzas del escenario político tuvieron incentivos para proponer un cambio de modelo de sistema electoral.

Fruto de la transformación del sistema de partidos catalán y habiendo trascendido a la opinión pública la posibilidad de un nuevo Estatuto de autonomía, se reactivó el debate de una posible una reforma electoral. Las organizaciones partidistas desempolvaron las propuestas que durante largos años han estado aparcadas y se inicia un periodo fecundo en la historia que estamos construyendo.

\section{El Estatuto de Autonomía de 2006 y los modelos de los partidos políticos}

En el momento en el que la competitividad electoral aumenta y el número efectivo de partidos políticos también, se abrió una posibilidad para la modificación del sistema electoral. Pero en el momento en el que se intuye esta posibilidad, todo había cambiado:

a) Desde el punto de vista institucional, el principal cambio que conlleva la aprobación del Estatuto de autonomía de Catalunya de 2006 es el artículo 52.2, en el que se establece que «(...) El régimen electoral es regulado por una ley del Parlamento aprobada en una votación final sobre el conjunto del texto por mayoría de dos terceras partes de los diputados». La reforma catalana, en este aspecto, no está al margen del resto de modificaciones de los estatutos de autonomía de principio de los años 2000, más bien el contrario: se observa una generalización de la rigidez para la aprobación y modificación de la legislación electoral ${ }^{39}$. En el caso catalán, este significativo cambio podía tener un cierto sentido en la

38 Colomer, J. M., Cómo votamos. Los sistemas electorales del mundo: pasado, presente y futuro, Barcelona, Gedisa, 2004, p. 112.

39 Presno, M., «Nuevos Estatutos de autonomía con viejos sistemas electorales», Revista d'estudis autonòmics i federals, $\mathrm{n}^{\circ}$ 5, 2007, p. 137. 
estructura bipartidista clásica del sistema de partidos catalán, pero es muy poco eficiente en el nuevo formato que nace a partir de los años 2000, no sólo por la propia dificultad del pacto multilateral sino porqué provoca la capacidad de veto de alguno de los actores. Veremos que el cambio de las reglas de juego electorales será decisivo en el último intento de pacto entre las fuerzas políticas catalanas.

En segundo lugar, el Estatuto de autonomía de Catalunya de 2006, establece en el artículo 83.1 que «Catalunya estructura su organización territorial básica en municipios y veguerías». A diferencia del derogado Estatuto de 1979, que ordenaba el territorio en municipios y comarcas, la nueva norma institucional sitúa la veguería al frente de la organización territorial de Catalunya, junto con la entidad local básica por excelencia, el municipio. La comparación entre las previsiones del Estatuto de 1979 y el de 2006 en relación a la organización territorial supramunicipal pone en evidencia un cambio de dirección significativo, en la medida en que el centro de atención se desplaza de la comarca a la veguería, sin que se plantee una sustitución institucional sino una cohabitación entre la diversidad de órganos. De hecho, entre el año 2006 y la Ley 30/2010, de veguerías, la Administración de la Generalitat se organiza para la prestación de sus servicios públicos en siete zonas territoriales que, en cierta medida, se corresponden con los límites vegueriales.

b) Coadyuvando la menor presencia de la comarca, entre la propia opinión pública catalana, la comarca había ido desapareciendo del imaginario colectivo catalán, como la entidad natural de organización territorial catalana. No existen demasiados datos muestrales que reflejen las actitudes de los ciudadanos frente a las comarcas y los consells comarcals, instituciones de gobierno de este nivel político administrativo. En el año 2012 las empresas Broadcaster y GESOP realizaron una encuesta cuyos resultados demuestran que son más los catalanes que no consideran necesarios los consells comarcals, incluso en el interior de Catalunya donde, debido al tamaño poblacional, éstos juegan un rol supramunicipal más importante.

c) Finalmente, desde el punto de vista demográfico, a lo largo de estos años la población de Catalunya ha sufrido un crecimiento de más del veinte por ciento, pasando de los casi seis millones de habitantes a los siete y medio; es decir, una ganancia de más de un millón y medio de ciudadanos. La dinámica de la población catalana muestra tres períodos bien definidos con ritmos anuales de crecimiento muy diferentes. En primer lugar, la etapa hasta el año 1996, caracterizada por un estancamiento demográfico, en que la población crece en valores muy reducidos (un 2,5\% acumulado). Un segundo momento, en el que se produce una ruptura respecto a la dinámica de estabilidad anterior y se anticipa el importante choque inmigratorio de la década siguiente. Y, finalmente, los años 2000 en los que se produce el $80 \%$ del aumento demográfico de estas cuatro décadas, presentando una tasa de crecimiento insólita en la dinámica poblacional de los países europeos más avanzados: durante la expansión económica tiene lugar un verdadero boom demográfico y los residentes en Catalunya crecen en un millón. Este 
incremento poblacional se ha repartido por todo el territorio pero, de manera agregada, el $60 \%$ se ha concentrado en la circunscripción de Barcelona y, más concretamente, en la conurbación de la ciudad condal ${ }^{40}$. A pesar de esta distribución, las circunscripciones en las que el crecimiento poblacional ha sido más evidente son las provincias de Girona y Tarragona. Sea como sea, la relación escaño/ número de habitantes por distrito electoral ha quedado notablemente modificada respecto el prorrateo del Estatuto de 1979.

A lo largo de estos años, pues, Catalunya se ha transformado profundamente. De lo que se trata es de establecer de qué manera esta evolución ha afectado — si lo ha hecho de alguna manera - a los modelos de sistema electoral de las diferentes formaciones políticas.

Para llevar a cabo este cometido, analizaremos los distintos modelos de sistema electoral elaborados por los partidos políticos. Concretamente, hemos circunscrito el análisis a las propuestas de Convergencia Democrática de Catalunya, el Partit dels Socialistes de Catalunya, Esquerra Republicana de Catalunya e Iniciativa per Catalunya-Los Verdes. De todos ellos — salvo en el caso de CDC—se trata de las propuestas de Disposición Transitoria relativa al sistema electoral que se presentaron para incluir en el nuevo Estatuto de autonomía de 2006. En el caso de CDC, se trata de la propuesta que presentó públicamente en octubre del año 2003.

De manera general, es interesante poner de relieve que las divergencias en lo que atañe a la magnitud de la asamblea, al sistema de atribución de los escaños, a la barrera legal y al tipo de candidatura son muy menores, mientras que los principales desacuerdos se sitúan en los límites y la magnitud de los distritos. No podía ser de otra manera: no sólo el grado de proporcionalidad y la representación del territorio dependen directamente de esta variable, sino que ésta siempre ha sido la piedra angular del pacto. Veámoslo más en detalle.

\section{a) La magnitud de la cámara}

Del abanico de cámaras autonómicas, Catalunya ocupa el antepenúltimo puesto, ya que elige un escaño por cada 39.000 electores. Esta cifra está muy por encima de las autonomías uniprovinciales y las demás autonomías históricas, como Galicia - 30.000 electores - o el País Vasco — con una proporción de 25.000 electores por diputado. En este contexto, podría parecer que Catalunya dispone de un cierto margen para ampliar el número de diputados de su Parlamento. Pero, esta perspectiva resulta frenada por el hecho de que la cámara catalana es la más numerosa de todas las cámaras autonómicas. No parece, pues, que

40 Alberich, J. «L'ús de l'espai. Transformacions territorials a l'Àrea Metropolitana de Barcelona», Papers, n 51, 2010, pp. 28-43. 
por la vía de la magnitud de la cámara exista un gran margen para introducir cambios. Y así parece que lo han entendido los partidos políticos catalanes, ya que en ninguna de las propuestas que han presentado a la opinión pública se han decidido a aumentar el número de diputados para mejorar la representatividad del parlamento, es decir, buscar una proporción más ajustada entre población y electores - y el equilibrio territorial ${ }^{41}$.

\section{b) La fórmula de atribución de los escaños y la barrera minima}

En un ejercicio de mimetismo del sistema electoral de las elecciones generales, todas las comunidades autónomas se han decantado por el método de Hondt ${ }^{42}$. En Catalunya, este mimetismo se mantiene intacto, a pesar de que Iniciativa per Catalunya se haya decidido por el sistema de cociente y resto más alta.

También la barrera mínima del 3\% de los votos válidos emitidos en cada una de las cuatro circunscripciones procede del sistema electoral español para el Congreso de Diputados. En las propuestas de nuevo sistema electoral, las formaciones políticas se han inclinado, de forma unánime, para mantener la actual barrera legal, a pesar de que las posibilidades que brindan el resto de comunidades autónomas son múltiples.

\section{c) La circunscripción electoral}

Como ya lo hemos adelantado, la circunscripción es el elemento central de las discrepancias entre las diferentes propuestas: el Partit dels Socialistes de Catalunya e Iniciativa per Catalunya se inclinan por la circunscripción única y, en cambio, Convergencia Democrática de Catalunya y ERC proponen la veguería como distrito electoral.

En cuanto al prorrateo de las diferentes propuestas, lógicamente, sólo nos podemos referir a los modelos convergente y republicano, ya que la propuesta contrincante es la circunscripción única. En este sentido, el reparto de los 135 escaños entre las 7 veguerías supone una magnitud media de 19 escaños, que la sitúa en una posición muy alejada de la media española para las elecciones generales, que es de 6,7 diputados por provincia. Sin embargo, la desviación típica demuestra que la distancia entre magnitudes es muy elevada, que van desde los 77/78 escaños de la veguería de Barcelona hasta los atribuidos a las zonas menos

41 Magre, J., «Reflexions...», op. cit., pp. 168-172.

42 Pallarés, F., «Los sistemas electorales de las Comunidades Autónomas. Aspectos institucionales», en J. Montabes, (ed.), El sistema electoral a debate. Veinte años de rendimientos del sistema electoral español, Madrid, Centro de Investigaciones Sociológicas/Parlamento de Andalucía, 1998, p. 237; LlerA, F. «Los rendimientos ...», op. cit., pp. 252-253. 
pobladas del Alto Pirineo o las Tierras del Ebro, que no superan los siete escaños.

En ninguno de los dos modelos se respeta la estricta proporcionalidad. El principio sobre el que se basa el prorrateo de ambas propuestas es, precisamente, lo que provoca el sesgo: en el caso de ERC, se propone una preasignación de cuatro escaños por distrito y el resto se distribuye entre las veguerías en proporción a su población. El sistema utilizado para CDC, en cambio, parte del prorrateo actual de base provincial y establece un nuevo reparto interno sobre la base de la población de cada una de las veguerías. En ambos casos - ya sea porque otorga un mínimo de diputados con independencia de la población o porque se mantiene el reparto actual - la distribución de los escaños prima de forma clara ciertas zonas de la geografía catalana. Y así debe ser. Lo que no parece prudente es que la veguería de Barcelona ocupe el 70 por 100 de la cámara catalana y que los cuarenta dos escaños restantes deban repartirse entre el resto de comarcas ${ }^{43}$.

Excepto en el caso de ERC, en el resto de propuestas se lleva a cabo un segundo reparto territorial. Esta distribución se establece con el fin de conseguir una representación más adecuada de las diferentes zonas de Catalunya y, por tanto, no tiene ninguna influencia en el grado de proporcionalidad que pueda alcanzar el sistema electoral. En este contexto, ICV y el PSC mantienen los límites provinciales para la distribución interna de los diputados a partir de fórmulas muy complejas desde el punto de vista técnico para conservar el prorrateo actual. $\mathrm{Y}$, con respecto al modelo convergente, se trata de un sistema mixto en el que las cuarenta y una comarcas y los diez distritos de Barcelona se convertirían en circunscripciones uninominales. Concretamente, el ciudadano tiene dos votos en una misma papeleta. Un primer voto a una lista cerrada y bloqueada de partido en su veguería - a partir del cual se asignarían los escaños en las diferentes candidaturas-, y un segundo voto a un candidato individual en su comarca o distrito, en el caso de Barcelona ciudad. De este modo, cincuenta cuatro escaños se elegirían en circunscripciones uninominales y el resto, hasta sumar el total de escaños asignados a cada partido político, serían ocupados por los candidatos de las listas presentadas en cada una de las veguerías.

Recapitulando, pues, existen razones para entender que las fuerzas políticas hayan convergido mínimamente, adaptándose a la nueva fisonomía de Catalunya $\mathrm{y}$, al mismo tiempo, ciertos detalles que nos indican la dificultad que supone alejarse de la presión de la opinión pública y el cálculo electoral por parte de las organizaciones partidistas:

a. Sólo CDC mantiene la comarca como circunscripción electoral de segundo nivel. Veintisiete años no han pasado en vano. En 1979 el frente comarcalista abarcaba a CDC, ERC UC-DCC y UCD. Existía entre estas fuerzas

43 Magre, J., «Reflexions...», op. cit., pp. 168-172. 
políticas un acuerdo tácito según el cual la circunscripción uninominal de base comarcal era la mejor estrategia para conseguir una buena representación del territorio en la cámara catalana. Las propuestas de Disposición Transitoria relativa al sistema electoral que se presentaron para incluir en el nuevo Estatuto de autonomía de 2006 certifican su abandono. Y este abandono es una buena noticia: la representación del territorio en el Parlamento no puede fundamentarse en las cuarenta y una comarcas, ya que cualquier proyección hecha a partir de esta circunscripción afecta a la propia racionalidad del sistema electoral.

b. La aplicación del prorrateo inicial de la Disposición Transitoria se hizo con los datos censales del año 1976 y no tiene sentido mantenerlo inalterado a pesar de los más del millón y medio de habitantes con los que se ha incrementado el padrón los últimos años. El incremento es especialmente visible -alrededor del $40 \%$ - en las provincias de Girona y Tarragona y, en cambio, en los distritos de Lleida y Barcelona se sitúa en menos del 20\%. Sea como sea, la intención de la mayoría de las formaciones de mantener la representación inicial de cada uno de los distritos, con independencia de la circunscripción que se proponga, tiene más que ver con la intención de no abrir la caja de pandora de la opinión pública que de un cálculo racional de los partidos políticos.

c. Las dos visiones ideológicas sobre el territorio y, en el fondo, sobre qué es Catalunya, se han debilitado. Los partidos otrora comarcalistas han derivado hacia la veguería como circunscripción y los partidos de izquierda apuestan por la circunscripción única: la geografía electoral de los partidos de izquierda y, sobretodo el PSC, determina el cambio hacia la circunscripción única. Los resultados de las elecciones autonómicas de 1999 y 2003 han demostrado a las formaciones de izquierda que, según en qué circunstancias e incluso ganando en número de votos, pueda no tener una equivalencia en el número de escaños. La única manera que esta situación no se reproduzca es apostando por la circunscripción única. A pesar de todo, es posible vislumbrar una cierta opción de convergencia de los partidos políticos hacia la veguería como circunscripción para las elecciones al Parlamento de Catalunya. Esta es la posibilidad a la que se aferra el Informe de los expertos que en 2007 intenta buscar un mínimo común denominador para hacer factible una propuesta de sistema electoral.

\section{LA COMISIÓN DE EXPERTOS DEL AÑO 2007 Y LA PONENCIA PARLAMENTARIA DEL AÑO 2010}

La única tentativa de carácter institucional que ha existido hasta la fecha para facilitar un pacto entre las fuerzas políticas, fue la creación por parte de la Generalitat de Catalunya de una Comisión de expertos para que elaborarse los 
trabajos previos a la redacción de la Ley electoral de Catalunya. Concretamente, en el Acuerdo de Gobierno de 27 de marzo de 2007 se establece que «El Gobierno ha considerado necesario crear esta Comisión antes de la ponencia conjunta en el Parlamento, para que los expertos en materia electoral elaboren los trabajos que servirán de punto de partida de la futura ley».

Los tres acuerdos centrales que se proponen en los documentos de trabajo de la Comisión son, en primer lugar, que los diputados se elijan en siete circunscripciones, correspondientes a los ámbitos territoriales en que está organizada la Administración de la Generalitat, que se ajustan a los límites vegueriales. En segundo lugar, la eliminación del actual número máximo de escaños para la circunscripción de Barcelona, así como la adjudicación de un mínimo inicial de dos escaños a cada una de las otras seis circunscripciones y reparto de los 123 restantes entre todas las circunscripciones según la población. Y, finalmente, la sustitución de la lista cerrada por una lista desbloqueada que permita el voto preferente a algunos candidatos individuales. Concretamente, se establece la elección prioritaria de los candidatos que hubieran obtenido un número de votos preferentes superior al 5\% de los votos de la candidatura. Por lo que atañe al resto de variables del sistema electoral, se opta por el mantenimiento de la fórmula de asignación de escaños a los partidos según la media más alta, así como la barrera mínima de un 3\% de los votos en cada circunscripción y la magnitud de la cámara catalana.

Los miembros de la Comisión, conscientes que las posiciones fundacionales de las organizaciones se habían diluido y habían perdido parte de su razón de ser, proponen la veguería como única solución posible o, como mínimo la más probable. En cierta medida, no deja de ser una solución propiciada por las circunstancias. Las reformas institucionales, los cambios demográficos y de cultura política podían ayudar al pacto político. No tenemos que olvidar que la Administración de la Generalidad ya se organizaba en siete zonas territoriales para la prestación de sobre Servicios Públicos que, en cierta medida se corresponde con los límites de las veguerías y el Estatuto introdujo, como ya se ha indicado, a la veguería como parte de la estructura territorial básica de la Generalitat.

Dicho de otra manera, la cosmovisión que estaba ligada, en los momentos fundacionales, a una cierta idea de Catalunya, ahora se había diluido lo suficiente como para encontrar una solución intermedia. La propuesta de la Comisión es un sistema que no se aleja en exceso del actual, en que se elimina el umbral máximo al que podía acceder la circunscripción de Barcelona, los 85 escaños. Esta decisión conlleva, lógicamente, una disminución de los diputados que se distribuyen, con independencia de la población, en cada uno de los distritos electorales. Se adapta, pues, el prorrateo inicial llevado a cabo a partir del censo de 1976 y se acepta que algunas de las circunscripciones pierdan representatividad en el Parlamento. Una cierta renuncia en la que las formaciones de izquierda y el Partido Popular abandonarían la idea de la circunscripción única y los partidos nacionalistas aceptarían romper con el techo de los 85 escaños del distrito electoral de Barcelona. 
En julio de 2009, dos años después la presentación pública del Informe de la Comisión, todos los grupos parlamentarios manifestaron la voluntad de impulsar una proposición de Ley electoral y con este cometido solicitaron la creación de una ponencia parlamentaria con representación de todos los partidos parlamentarios para elaborarla a partir del documento de la Comisión de expertos Participación, representación, transparencia. Informe para la Ley electoral de Catalunya. La Ponencia conjunta se llevó a cabo el año 2010.

La única información de la que disponemos es la documentación anexa al Informe que aportan todos los partidos, ya que se acordó que las sesiones de la Ponencia no se grabarían ni se transcribirían, salvo las comparecencias de expertos (Informe de la Ponencia de la Comissió d'Afers Institucionals. Butlletí Oficial del Parlament de Catalunya, 7 de mayo de 2010, núm. 701, p. 3). La información al alcance es, pues, limitada y no nos permite conocer los términos del debate. Lo que sí sabemos con seguridad es que los trabajos preparatorios a la ponencia realizados por la Comisión de expertos han servido de muy poco, ya que las posiciones de las distintas formaciones políticas se mantienen prácticamente intactas. Respecto a la circunscripción, ERC y CiU continúan apostando por la veguería y con un segundo nivel comarcal en el caso de los convergentes. Por el contrario, PSC, ICV y el Partido Popular reinciden en la propuesta de circunscripción única. ${ }^{44}$ Respecto al prorrateo, aún peor: ERC incrementa el reparto inicial a los distritos de dos a tres escaños cada uno y CiU insiste en respetar lo que han venido a llamar el «prorrateo fundacional», es decir, mantener la distribución de escaños actual, a pesar de los cambios demográficos a los que hemos hecho referencia (Informe de la Ponencia de la Comissió d'Afers Institucionals. Butlletí Oficial del Parlament de Catalunya, 7 de mayo de 2010, núm. 701, p. 251).

Y no se trata de una estrategia de negociación, se trata de la incapacidad de llegar a un acuerdo. La ponencia estableció en sus conclusiones que «acuerda comunicar a la Mesa del Parlamento que no puede elaborar, de conformidad con lo que establece el artículo 117.1 del Reglamento, una proposición de ley electoral de Catalunya, de carácter general o sectorial que, en la votación final, pueda conseguir el apoyo de dos terceras partes de los miembros del Parlamento, exigido por el artículo 56.2 del Estatuto» (Informe de la ponencia de la Comisión d'Afers Institucionals. Butlletí Oficial del Parlament de Catalunya, núm. 701, 7 de maig de 2010). En efecto, se trata de la confirmación de la impotencia ya que, tal y como dejan entrever las conclusiones, la mayoría requerida para la aprobación de la Ley electoral catalana dificulta sobremanera la posibilidad de un pacto entre las fuerzas políticas y, ante tal escenario, reaparecen las posiciones maximizadoras de cada una de ellas. Lo que pretendía el artículo 56.2 del Estatuto de 2006 que

44 Dejamos de lado las aportaciones del representante de Ciudadanos ya que esta formación no ha formado parte del universo del estudio. Por otro lado, es la única organización que apostaría por el mantenimiento del sistema actual preasignando un único diputado por circunscripción y eliminando el tope de los 85 escaños en el distrito de Barcelona. 
no era nada más que la obligación de buscar un gran acuerdo político para la ley electoral, se ha convertido en un lastre para la capacidad de maniobra de los partidos políticos que, de momento, han renunciado a un nuevo intento.

\section{CONCLUSIONES}

Lo que hemos pretendido con este trabajo es hacer un recorrido por las propuestas de sistema electoral realizadas por los distintos partidos políticos a lo largo de cuatro décadas de sistema político catalán para acabar mostrando que, aun siendo posible el pacto, la rigidez de la reforma del nuevo Estatuto lo ha imposibilitado.

El artículo ha estudiado tres situaciones —que coinciden con la elaboración de los dos estatutos de autonomía y la Comisión de expertos creada por Acuerdo de gobierno el año 2007- que nos permiten poner a prueba la path dependency de las diferentes fuerzas políticas respecto del primer diseño que realizan durante los primeros años de la transición política.

El primer momento, se centra en la Comisión de los veinte y el Pleno de los parlamentarios durante la redacción y aprobación del Estatuto de 1979. El desacuerdo central en los debates fue la circunscripción y, más concretamente, el papel de la comarca en el diseño del sistema electoral. Más allá de los evidentes intereses electorales de las formaciones políticas, el trasfondo nacionalista de este ente territorial dificultó de gran medida el pacto de la Disposición Transitoria cuarta. Se acabó acordando la provincia porque no existía una alternativa consensuada entre dos mundos muy distintos en la manera de entender Catalunya y por la percepción compartida de los ponentes sobre la necesidad de llevar a cabo las primeras elecciones sin demora. La Comisión de los veinte estableció el camino del posible acuerdo: se desproporcionalizaba la representación a favor de las provincias menos pobladas a cambio de renunciar a la comarca como distrito electoral. Aun así, la comarca resistió y continuó siendo el caballo de batalla de los partidos nacionalistas durante muchos años.

La segunda etapa (1980-2006) es el intervalo en el que el sistema de partidos catalán se estabiliza y queda estructurado como un particular bipartidismo interelecciones. A lo largo de este período, los resultados producidos por el sistema electoral catalán se demuestran aceptables en términos de proporcionalidad, es decir, de la relación entre votos y escaños. Pero la diferente presencia de los partidos en el territorio provoca una distorsión que siempre ha jugado a favor de una fuerza política, Convergència i Unió, en una proporción que oscila entre los 3,2 puntos y 6,5 puntos porcentuales, según las legislaturas. Como resulta lógico, ateniendo a estas magnitudes, mientras ésta formación política estuvo en el gobierno de la Generalitat, el cambio de sistema electoral era una hipótesis difícil de concretar. En el momento en el que la competitividad electoral aumenta y el número efectivo de partidos políticos, también, se abre una posibilidad para la 
modificación del sistema electoral. Pero en el momento en el que aparece esta opción, todo ha cambiado: desde el punto de vista institucional, el nuevo Estatuto consagra las veguerías y establece una mayoría cualificada para aprobar la normativa electoral, desde el punto de vista de la opinión pública, la comarca ha ido desapareciendo del imaginario colectivo catalán y, finalmente, desde el punto de vista demográfico, la población ha sufrido los cambios suficientes como para poner en duda el prorrateo del Estatuto de 1979.

Atendiendo a este nuevo escenario, las fuerzas políticas habían de adaptarse. $\mathrm{Y}$ así lo hacen, en cierta medida: los partidos de tradición comarcalista derivan hacia la veguería como circunscripción, lo que deja intuir un posible pacto, que se concretará en el Informe de la Comisión de expertos del año 2007. La propuesta supone eliminar el tope máximo de los 85 escaños en la circunscripción de Barcelona, lo que conlleva una disminución de los diputados que se distribuyen, con independencia de la población, en cada uno de los distritos electorales. Se adapta, pues, el prorrateo inicial llevado a cabo a partir del censo de 1976 y se acepta que algunas de las circunscripciones pierdan representatividad en el Parlamento.

A partir de este momento, las reformas institucionales del Estatuto del año 2006 incentivan las posiciones maximizadoras de los partidos políticos y entorpecen un pacto que las circunstancias del momento habían favorecido. La rigidez de la aprobación de la ley electoral ha reabierto la distancia entre las fuerzas políticas y aumenta su capacidad de veto.

$$
* * *
$$

\section{TiTLE: Forty Years of the Electoral Law for the Parliament of Catalonia}

Abstract: Despite the many years that have elapsed since the adoption of the Sau Statute, in 1979, the electoral law has not been approved and Catalonia. It is the only autonomous community without its own electoral law. The article makes an itinerary between the different proposals of the political formations through four decades of the Catalan political system - from the three moments in which the parties present their electoral system models - to end up showing that the rigidity of the reform of the 2006 Statute regarding the approval of the Electoral Law, it has encouraged the maximizing positions of the political parties and has accommodated the veto positions, which has reduced the negotiation and the possibilities of agreement.

Resumen: A pesar de los muchos años transcurridos desde la aprobación del Estatut de Sau, el año 1979, la prevista ley electoral no se ha llegado a aprobar y Catalunya se ha convertido en la única comunidad autónoma sin una ley electoral propia. El artículo realiza un itinerario entre las distintas propuestas de la formaciones políticas a través de cuatro décadas de sistema político catalán - a partir de los tres momentos en los que los partidos presentan sus modelos de sistema electoral - para acabar mostrando que la rigidez de la reforma del Estatuto de 2006 en lo que atañe a la aprobación de la Ley electoral, ha incentivado las posturas maximizadoras de los partidos políticos y ba dado cabida a las posiciones de veto, lo que ha restado eficacia a la negociación y a las posibilidades de pacto.

KEY WORDS: Electoral system, comarca, vegueria, territorial organization, political parties

PALABRAS Clave: Sistema electoral, comarca, veguería, organización territorial, partidos políticos

FECHA DE RECEPCIÓN: 30.01.2020

Fecha de ACEPTACión: 19.02.2020 
